\title{
Synthesis of Nonlinear Observers for Actuator Fault Detection and Isolation
}

\author{
Rania Loukil \\ Control and Energy management \\ Laboratory, National Engineering \\ School of Sfax, University of Sfax. \\ Tunisia.
}

\author{
Mohamed Chtourou \\ Control and Energy management \\ Laboratory, National Engineering \\ School of Sfax, University of Sfax. \\ Tunisia.
}

\author{
Tarak Damak \\ Industrial Processes Control Unit, \\ National Engineering School of \\ Sfax, University of Sfax. Tunisia.
}

\begin{abstract}
In this works, we use the approach based on observers such as the Luenberger observer and the sliding mode observer in order to introduce the diagnosis of nonlinear systems. The robustness of the proposed observers is tested through a physical example. The obtained results show that for non linear systems the performances of sliding mode observer observer is better than using a classic kind of observer. The synthesis of nonlinear observers will be used for actuator fault detection and isolation using residual generation. Finally, a comparison of observers' performances will be interesting for judging the effectiveness of this approach.
\end{abstract}

\section{Keywords}

Observers, nonlinear systems, Luenberger observer, Sliding mode observer, fault detection and isolation, residual generation.

\section{INTRODUCTION}

The main objective of an automatic control system is to assure an acceptable performance which cannot be achieved only by improving the individual reliability of the functional units. It also requires an efficient fault detection, isolation and accommodation capability. Several conventional linear as well as nonlinear observers have been suggested during the past decades, so different techniques of testing the observability of nonlinear system are presented in literature. For this reason many observability analysis are developed for these systems to judge the nonlinearity. An observer is a copy model with a term of correction which establishes the convergence of the estimate state to the real state. It's called also a software sensor. In literature, the observers developed for the nonlinear systems differ from those of linear ones. In this paper we study a Luenberger observer [6] and a sliding mode observer [3]. Then we develop them and we formulate diagrams for simulation. Besides, the performances of the proposed observers are tested through a physical example. Finally, we present an approach for fault diagnosis of the same nonlinear system and we define the actuator fault detection and isolation using the different kind of observers. A comparison study will be interesting for judging the importance of the used method.

\section{SYNTHESIS OF NONLINEAR OBSERVERS}

The observer which is described in fig 1 is a useful tool used for estimation and failure diagnosis especially for monitoring based on models and in this sense we will use different type of observers in order to calculate the observed error and compare between different methods in order to improve the performances.

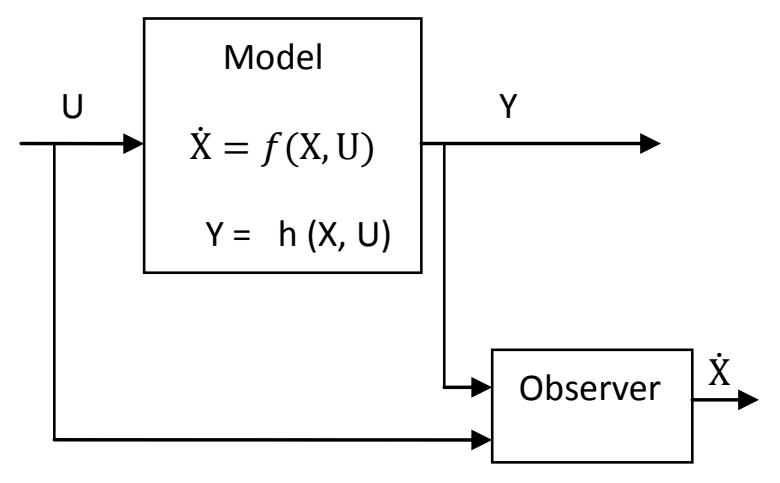

Fig. 1. Synthesis of nonlinear observer

\subsection{Synthesis of a Luenberger observer}

In 1964, Luenberger introduced his well-known state estimator for linear and nonlinear systems [6].

The Luenberger observer is indeed the simplest type of the closed-loop observers. In this observer, the output error will be fed back to the observer. This gain is selected such that $(A-L C, C)$ be observable. Consider the following state space representation:

$$
\left\{\begin{array}{l}
\dot{x}(t)=A x(t)+B u(t) \\
y(t)=C x(t)
\end{array}\right.
$$

With $\mathrm{A}, \mathrm{B}$ and $\mathrm{C}$ are nonlinear matrix, $\mathrm{x}$ is the state and $\mathrm{u}$ is the input. Then the structure of the Luenberger observer is given by:

$$
\left\{\begin{array}{l}
\dot{\hat{x}}(t)=A \hat{x}(t)+B u(t)+L(y(t)-\hat{y}(t)) \\
\hat{y}(t))=C \hat{x}(t)
\end{array}\right.
$$


This observer can also be used for the plant with deterministic noises we define $\mathrm{L}$ as a positive gain.

\subsection{Sliding mode observer}

It provides robust performance for observing the states of nonlinear systems with uncertainties. It is well known that modeling inaccuracies can have strong adverse effects on nonlinear observer systems.

Therefore, any practical design must address them explicitly. Two major and complementary approaches to dealing with model uncertainty are robust and adaptive approaches. In fact, the sliding mode observer was shown successful in dealing with model-free systems [2].

The idea is to define a time-varying surface $(s(x, t))$ in the state-space such that the problem of $\mathrm{s}=x(t)-\overline{x(t)}$ is equivalent to remain on the surface $s(t)$ for $\mathrm{t}>0$ [13]. The major difficulty here is how to find a suitable sliding surface $(s(t))$. To design a sliding mode observer, let us consider the general state space model:

$$
\left\{\begin{array}{l}
\dot{x}=f(x, u) \\
y(t)=c x(t)
\end{array}\right.
$$

Where $x \in R^{n}$ is the state vector, $u \in R^{m}$ is the bounded control input, and $C$ is known. Then the structure of the Sliding mode observer is given by:

$\dot{\hat{\mathrm{x}}}(\mathrm{t})=\hat{\mathrm{f}}(\mathrm{x}, \mathrm{u})+\lambda \operatorname{sign}(\mathrm{s}(\mathrm{t}))$

We choose $\lambda>0$ and properly fixed for the estimation and the diagnosis [12].

\section{CASE OF TWO LEVEL TANKS}

The system of two- level tanks presented in figure 2 consists of a set of elementary components : the top tray $(\mathrm{BH})$, the pan bottom (BB), a valve gain $\mathrm{K}_{1}$ from the top ( $\mathrm{VKH}$ ) and a valve gain $\mathrm{K}_{2}$ from the bottom (VKB), different sensors that allow us to measure the height of the product in the tank top $\left(\mathrm{H}_{1}\right)$ and the tank bottom $\left(\mathrm{H}_{2}\right)$ and the inflow of the product $\left(\mathrm{q}_{\mathrm{e}}\right)$ and $\left(\mathrm{q}_{1}\right)$ in the tank top and the bottom tank respectively. The flow admission is controlled directly (open loop) by a digital computer.

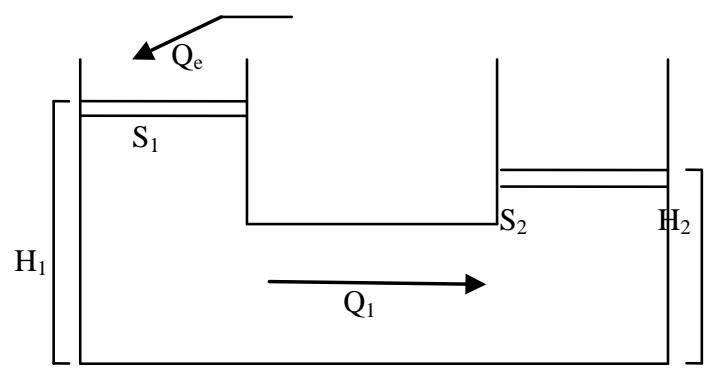

Fig.2. Description of the nonlinear system under study
The dynamic model of the system can be presented as follows:

$S_{1} \frac{\mathrm{dh}_{1}}{\mathrm{dt}}=\mathrm{q}_{\mathrm{e}}-\mathrm{q}_{1}$

$S_{2} \frac{\mathrm{dh}_{2}}{\mathrm{dt}}=\mathrm{q}_{1}-\mathrm{q}_{\mathrm{s}}$

$\mathrm{q}_{\mathrm{s}}=\mathrm{k}_{1} \sqrt{\mathrm{h}_{1}}$

$\mathrm{q}_{1}=\mathrm{k}_{2} \sqrt{\mathrm{h}_{1}-\mathrm{h}_{2}}$

\section{SIMULATION RESULTS FOR ESTIMATION}

In this section we apply the proposed methods to our disturbed system described previously. The main objective here is firstly to demonstrate the procedure of different observers required to achieve identification or diagnosis, secondly is to generate residuals and compare the performances of the different observers. In the simulation of our system, we apply the same flow $\mathrm{q}_{\mathrm{e}}$ for the three observers.

\subsection{Estimation results using a classic observer}

Using the classic observer, we obtain after the matlab simulation the flow $\mathrm{q}_{\mathrm{e}}$ which is the input of our system (fig.3) with $\mathrm{L}=0.19$.

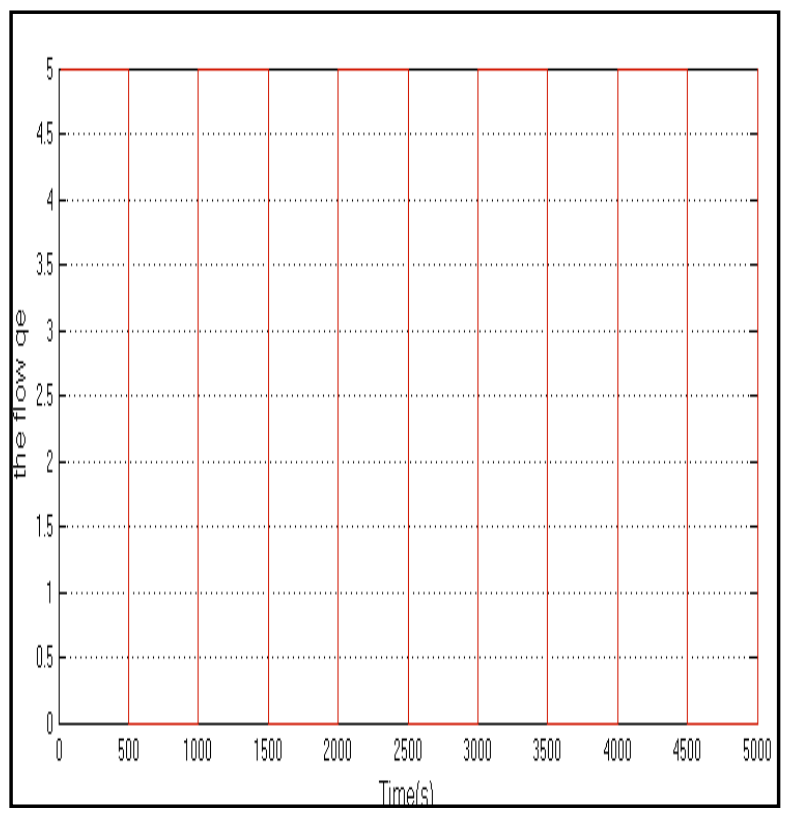

Fig3. The varying flow $q_{e}$

We obtain the height $h_{1}$ and its estimated $\hat{h}_{1}$ using the Luenberger observer (fig 4.a) and the observed error $e_{1}$ between them (fig 4.b). 


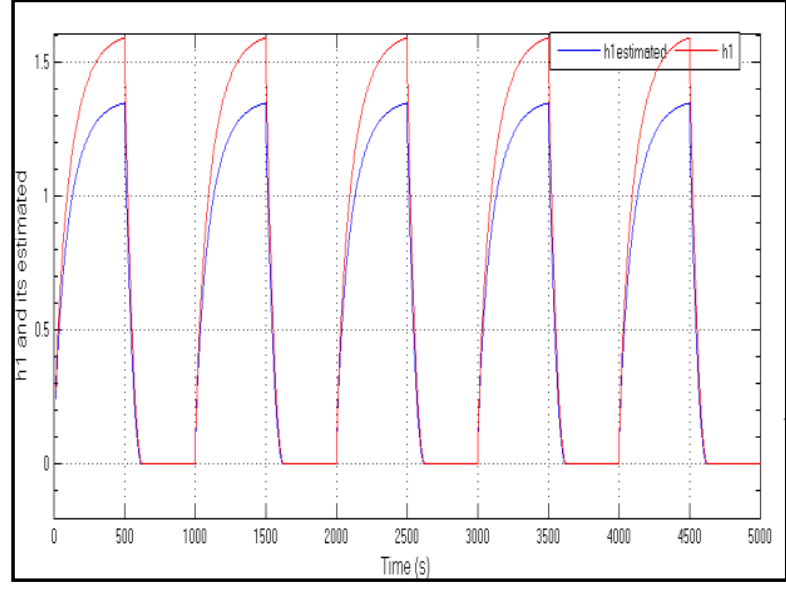

Fig.4.a. The height $h_{1}$ and its estimated

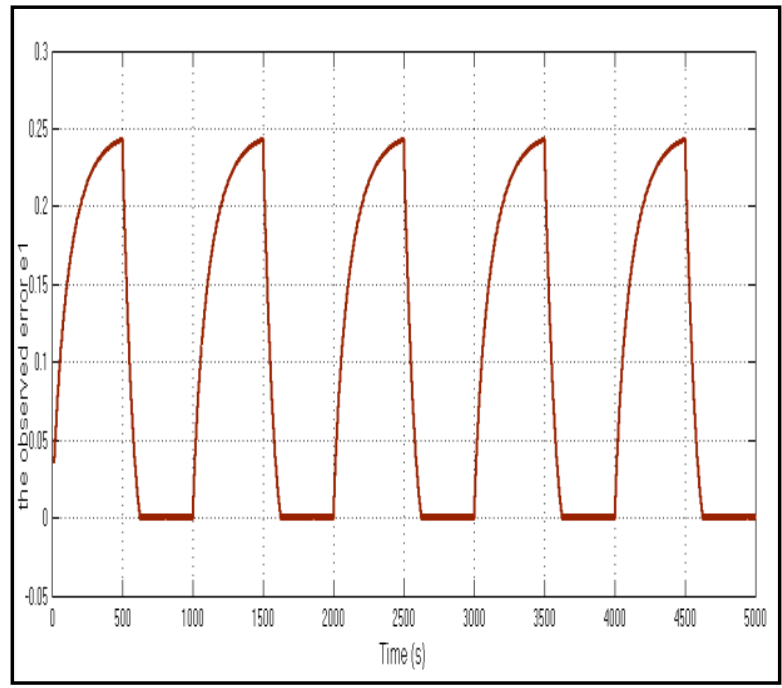

Fig.4.b The observed error $e_{1}$

We find also the height $\mathrm{h}_{2}$ and its estimated $\hat{h}_{2}$ (fig 4.c) and the observed error $e_{2}$ (fig 4.d) which must be implemented in order to evaluate the performance of this kind of observer.

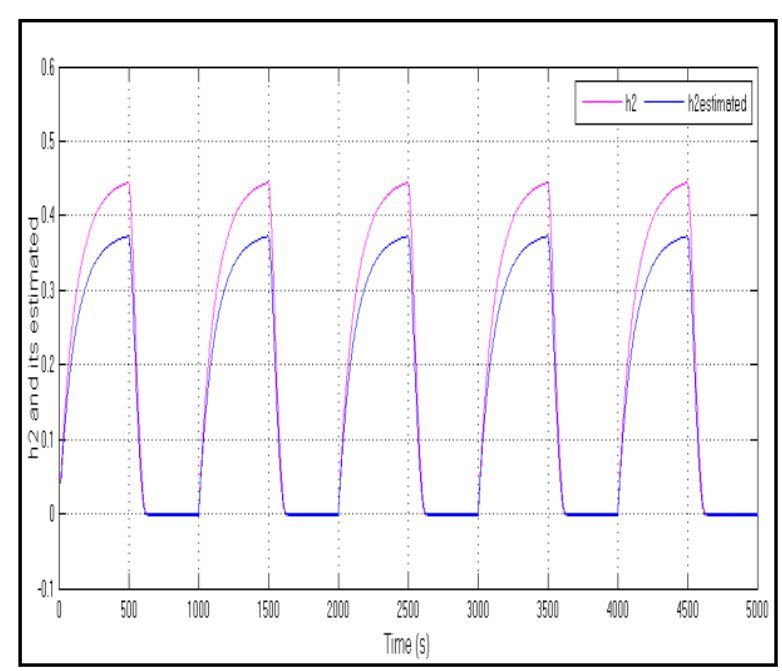

Fig.4.c. The height $h_{2}$ and its estimated

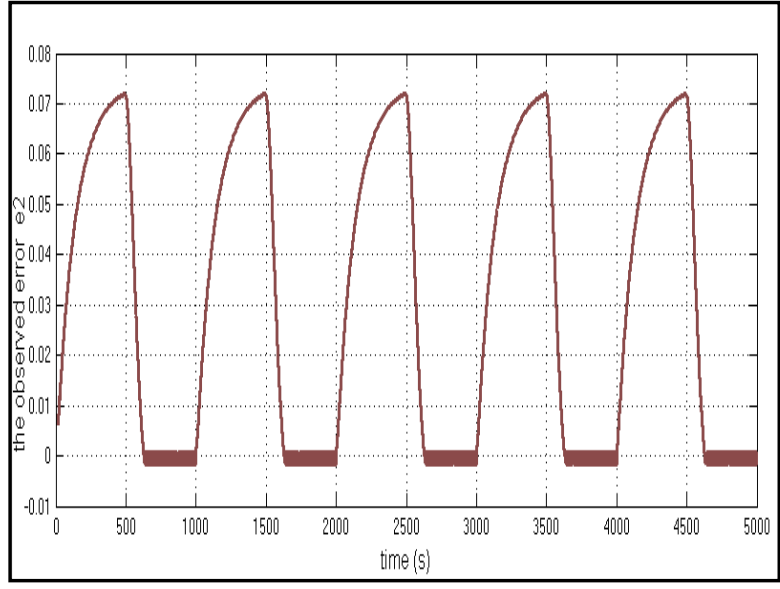

Fig.4.d. The observed error $e_{2}$

\subsection{Estimation results using a sliding mode observer [8]}

To compare the performance of the observers, we simulate the observed error $e_{1}$ between $h_{1}$ and its estimated $\hat{h}_{1}$ then the observed error $e_{2}$ between $h_{2}$ and its estimated $\hat{h}_{2}$ so we obtain (fig 5.a and 5.b).

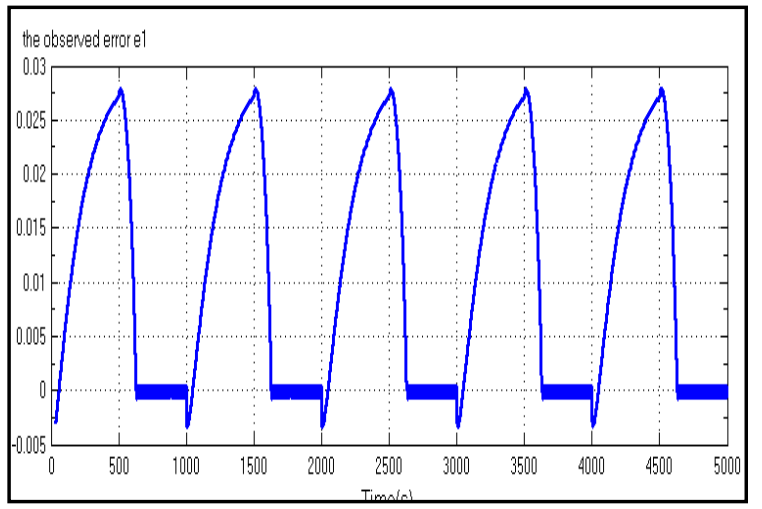

Fig.5.a. The observed error $\mathrm{e}_{1}$

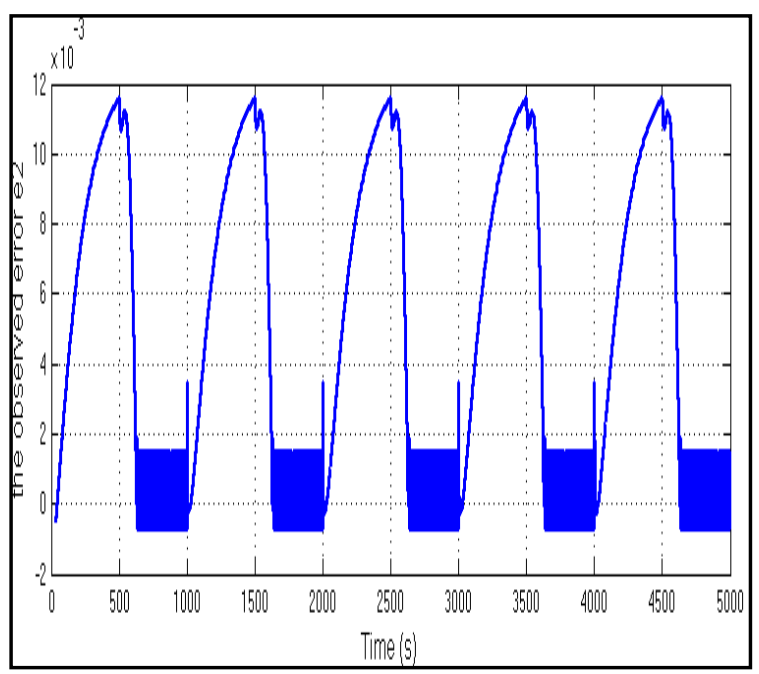

Fig.5.b. The observed error $e_{2}$ 


\section{SIMULATION RESULTS FOR DIAGNOSIS}

In this paper, we assume that only actuator faults can occur and no sensor faults are involved. For simplicity, we consider the case that only one single actuator is faulty at one time because extensions to multi-faults situation are straightforward. During this part, we will use a specific algorithm for the FDI scheme [4] which is composed of six steps:

* We form the new faulty model

* We build a bank of m observers for detection and isolation of fault.

* We generate the residuals $\mathrm{r}_{\mathrm{i}}(\mathrm{t})$.

* From the thresholds $\delta_{\mathrm{i}}$ and the fault code $\varepsilon_{\mathrm{j}}(\mathrm{t})$ we elaborate the structure matrix $\Phi$.

* We generate the structured residuals for the fault isolation and identification.

* We estimate finally $f_{\text {ai }}$

To do this, we will determine the appropriate thresholds $\delta_{\mathrm{i}}=$ constant and build the vectors of fault codes $\varepsilon_{j}(t)$ composed of $r_{i}(t)[1]$.

$\mathrm{r}_{\mathrm{si}}=\left[\begin{array}{l}r_{s 1} \\ r_{s 2} \\ r_{s 3} \\ r_{s 4}\end{array}\right]=\left[\begin{array}{llll}1 & 0 & 0 & 0\end{array}\right]\left[\begin{array}{l}f_{a 1} \\ f_{a 2} \\ f_{a 3} \\ f_{a 4}\end{array}\right]$

$\mathrm{a}_{\mathrm{i}}(\mathrm{t})=\left\{\begin{array}{l}1 \text { if }\left|r_{i}(t)\right| \geq \delta_{i} \\ 0 \text { if }\left|r_{i}(t)\right|<\delta_{i}\end{array}\right.$

$\varepsilon_{j}(\mathrm{t})=\left(a_{1}(t) a_{2}(t) \ldots a_{i}(t)\right)^{\mathrm{T}}$

$r_{i}=R\left(\hat{y}_{i}-y\right), i \in m$

We use $\mathrm{R}$ as an operator of the residual and in our case it is the norm 2 defined as the difference between the estimated output and measured output, so (8) becomes:

$$
r_{i}(t)=\left\|\hat{y}_{i}-y\right\|, \mathrm{i}=1, \ldots, \mathrm{m}
$$

In fact in our case, comparing the observed error of the sliding mode observer to the classic one, we find that the first one is useful for the estimation. In fact, $\mathrm{e}_{1 \max }$ is equal to 0.027 and $e_{2 \max }$ is equal to $11.10^{-3}$, so that they are near to zero. Furthermore, concerning the Luenberger observer $\mathrm{e}_{1 \max }$ is equal to 0.24 and $\mathrm{e}_{2 \max }$ is equal to 0.07 .

For the isolation and identification of the fault, we must define the threshold $\delta_{i}, \mathrm{i}=1, \ldots, \mathrm{m}$ as adaptive because of the error measurement [10].

$\delta_{i}=\left|r_{i}(t-\tau)\right|+1$

We fix $\tau=4$ [11].

$\Phi=\left[\varepsilon_{1}(\mathrm{t}) \varepsilon_{2}(\mathrm{t}) \ldots \varepsilon_{\mathrm{m}}(\mathrm{t})\right]$ $\mathbf{r}_{\mathrm{s}} \leftarrow \Phi_{\mathrm{f}}$

\subsection{Diagnosis results using a classic Luenberger observer}

The figure 6.a shows the variation of the residual $r_{1}$ during the time using a classic observer which is the Luenberger one. We find that $r_{1}$ has a positive peak at $t_{f}=20$ s the time of actuator fault injection, but it doesn't converge rapidly to zero. We choose a gain $\mathrm{L}_{1}=0.19$ [14].

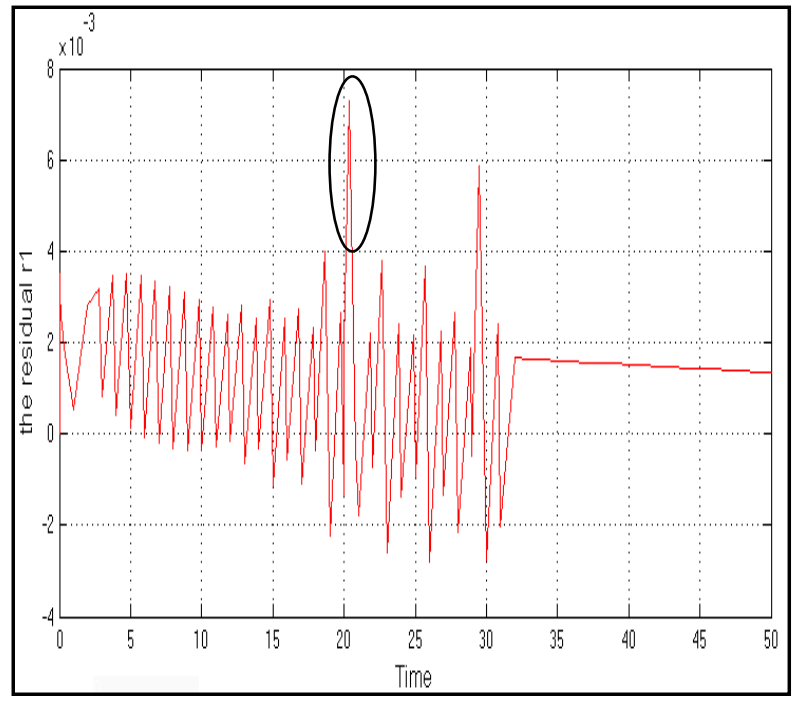

Fig.6.a. The residual $\mathbf{r}_{1}$ using a classic observer

The figure 6.b shows the variation of the residual $r_{2}$ during the time using a classic observer. We find that $r_{2}$ has a negative peak at $t_{\mathrm{f}}=20 \mathrm{~s}$ the time of actuator fault injection, but it converge rapidly to zero comparing to $r_{1}$. We choose a gain $\mathrm{L}_{2}=0.19$

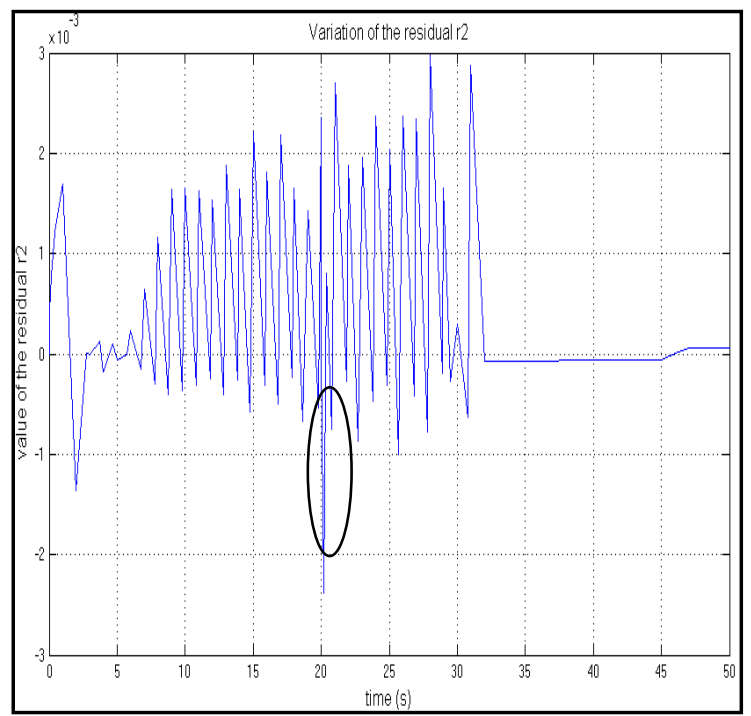

Fig.6.b. The residual $\mathbf{r}_{2}$ using a classic observer 


\subsection{Diagnosis results using a Sliding}

\section{mode observer}

In fact we have chosen $\mathrm{H}$ as a Hurwitz matrix so that the observer has a dynamics faster than the system. $\gamma$ is a constant and $\mathrm{P}$ is a design positive definite matrix solution of Lyapunov equation which is $\mathrm{H}^{\mathrm{T}} \mathrm{P}+\mathrm{PH}=-\mathrm{Q}$ [1]. We should notice that $\mathrm{Q}$ is a definite positive matrix, which can be chosen freely. For the simulation, the values of $\mathrm{H}, \mathrm{P}$ and $\gamma$ used in equation (7) are given: $\mathrm{H}=-5 \mathrm{I}_{3}, \mathrm{P}=\mathrm{I}_{3}$ and $\gamma=2$ and the injection of a single fault $f_{a 1}$ equal to $2 \mathrm{~m}^{3} / \mathrm{s}$ and at $t_{f}$ $=20 \mathrm{~s}$. The figure 8.a and 8.b show the variation of the residuals especially after the fault injection. However, if we have multiple faults injection on the system we move to the use of structured residuals (equation11).

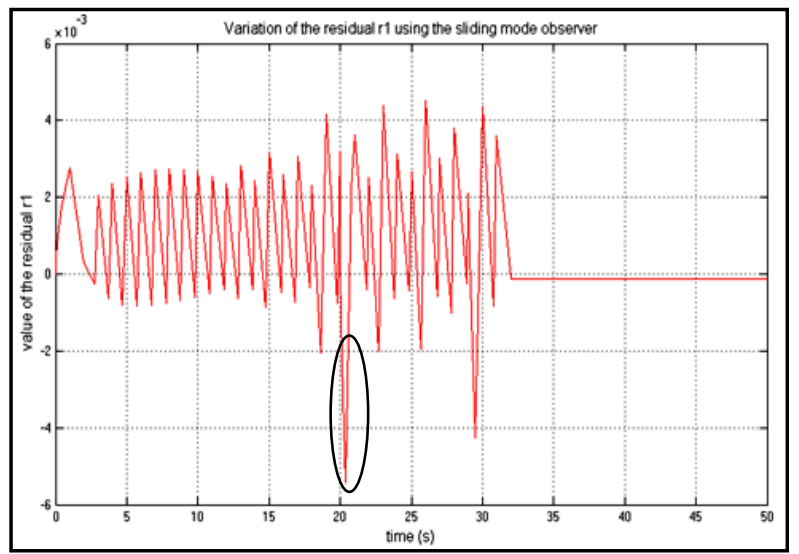

Fig.7.a. The residual $\mathbf{r}_{1}$ using a sliding mode observer

The figure 7.a shows the variation of the residual $r_{1}$ during the time using a sliding mode observer. We find that $r_{1}$ has a negative peak at $t_{f}=20$ s the time of actuator fault injection, and it converge rapidly to zero.

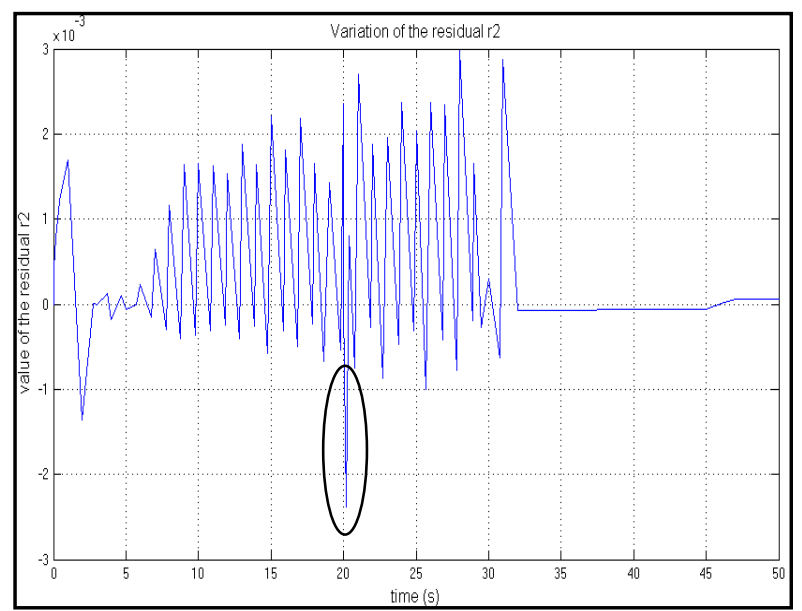

Fig.7.b. The residual $\mathbf{r}_{2}$ using a sliding mode observer

The figure $7 . b$ shows the variation of the residual $r_{2}$ during the time using a sliding mode observer. We find that $r_{2}$ has a negative peak at $t_{f}=20$ s the time of actuator fault injection and it converge rapidly to zero different from the classic one.

\subsection{Comparison of performances}

For the comparison of performances, we define: $t_{f}$ : fault occurrence moment, $t_{D}$ : fault detection moment, $t_{I}$ : fault isolation moment and $t_{R}$ : fault reset moment [15].

The target of this study is the comparison between the Luenberger observer and the sliding mode observer in order to validate our method (second one); in fact table 1 illustrate this when we compare the error of estimation, the time of the fault detection $t_{I}$ and the convergence speed [16].

Here, we find that the Luenberger observer has a minimum error of estimation but the sliding mode observer is more useful for the diagnosis. In fact, the amplitude of the residuals is inferior to the classic observer and we have estimated the fault as $\widehat{f_{a 1}}=2.25 \mathrm{~m}^{3} / \mathrm{s}$. Table1gives the different performances for analyzing an observer. Criterions such as the error of estimation, the amplitude of the residuals and the convergence speed can judge the effectiveness of the used observer.

Table.1. Analysis of observers' performances

\begin{tabular}{lccc}
\hline Oerformances & $\begin{array}{c}\text { Minimum } \\
\text { of Error } \\
\text { estimation }\end{array}$ & $\begin{array}{c}\mathrm{t}_{\mathrm{I}} \text { time of } \\
\text { The } \\
\text { fault } \\
\text { isolation }\end{array}$ & Speed \\
\hline $\begin{array}{l}\text { Luenberger } \\
\text { Observer }\end{array}$ & $\min$ & $\max$ & $\min$ \\
\hline $\begin{array}{l}\text { Sliding } \\
\text { Mode } \\
\text { observer }\end{array}$ & $\max$ & $\min$ & $\max$ \\
& & & \\
\end{tabular}

\section{CONCLUSION}

During this work, we have presented two kind of nonlinear observers which are the classic Luenberger observer and the sliding mode observer.

Through, the example of a nonlinear system named the two level tanks which was described previously, we have tested the robustness of the proposed observers in the estimation task.

Besides, we move to the diagnosis and actuator fault detection and isolation for the same system.

We describe the approch and its steps then we inject at $t_{\mathrm{f}}=20 \mathrm{~s}$ a fault of $2 \mathrm{~m}^{3} / \mathrm{s}$ and we discuss the variation of the residuals $r_{1}$ and $r_{2}$ during the time, their peaks and their convergence to zero.

Finally, a comparison study was done to judge the useful observer for estimation and diagnosis field.

Other kind of observers can be used in the same approch in order to validate this method such as the neural observer [7].

\section{REFERNCES}

[1] R. Loukil, M. Chtourou and T. Damak "An actuator fault detection and isolation for a nonlinear system using observers", International conference on Sciences and Techniques of Automatic control and computer engineering, Sousse, Tunisia. December 18- 20 (2011). 
[2] C.Weitian; J.Guangqing and S.Mehrdad "Application of sliding mode observers for actuator fault detection and isolation of linear system", IEEE conference on control application, Toronto, Canada, August 28-31 (2005).

[3] H.Saadaoui ,De leon ,M.djemai,J.P Barbo "High order sliding mode and adaptive observers for a class of switched systems with unknown parameter: A comparative study'. Proceeding of the 45th IEEE conference on decision and control san diego ,CA , USA. December 13-15 (2006).

[4] Dimitrios Frangkoulis. (2008). Phd thesis from the university of Toulouse III- Paul Sabatier, Toulouse, France. "Detection et isolation des défauts provenant des actionneurs et des capteurs: Application sur un système non linéaire“.

[5] A. Hafaifa, F.Laaouad et K. Laroussi,'Utilisation de la méthode FDI pour la localisation de défauts de fonctionnement d'un processus non linéaire, 4th International Conference on Computer Integrated Manufacturing CIP'2007.

[6] Luenberger D. G., 'An introduction to observer', IEEE transactions on, Automatic control, 16(6), pp. 596 602, 1971.

[7] R. Loukil , M. Chtourou and T. Damak "Training a neural observer using a hybrid approach ", IEEE conference on Systems, Signals \& Devices, Chemnitz, Germany. March 20-23 (2012).

[8] C.Edwards; S. K. Spurgeon and R. J. Patton "Sliding mode observers for fault detection and isolation", Elsevier Science Ltd. (2000).
[9] K.Boon Goh; S. K. Spurgeon and N. Barrie Jones "Fault diagnosis using sliding mode techniques", Elsevier Science Ltd. (2001).

[10] Damak, T. (2006) Contribution à la modélisation et à l'analyse de systèmes et au développement d'approches non linéaire d'estimation et de commande : Habilitation universitaire ENIS Sfax, Tunisie.

[11] Damak, T. (1994) modélisation estimation et commande des procédés biotechnologiques de type hyperbolique, Thèse de doctorat de l'université Paul Sabatier ; LAAS/CNRS ; Toulouse, France.

[12] Isermann, R. (1997).Supervision, fault-detection and fault-diagnosis methods-an introduction. Control Eng. practice, Vol.5, No 5, pp639-652.

[13] Isermann, R., \& Ballé, P. (1997). Trends in the application of model-based fault detection and diagnosis of technical processes" Control Eng. practice, Vol.5, No 5, pp709-719.

[14] Hammouri, H., Kinnaert, M., \& El Yaagoubi, E. H. (1999). Observer based approach to fault detection and isolation for nonlinear systems. IEEE Transactions on Automatic Control, 44, 10, pp. 1879-1884.

[15] Li, Z., \& Boutaieb, D, (2007). Fault isolation for nonlinear dynamic systems based on parameter intervals. International Journal of systems Science Vol, 38, $N^{\circ}$. 7, July, pp 531-547.

[16] Li, Z., \& Boutaieb, D, (2008). A new fault isolation and identification method for nonlinear dynamic systems: application to a fermentation process. Science Direct Applied mathematical Modelling 32, pp 2806-2830. 